\title{
Does Preoperative Sinus Rhythm Influence Surgical Ablation's Perioperative Safety in Patients with Atrial Fibrillation?
}

\author{
Maximilian Vondran, MD, ${ }^{1,2}$ Tamer Ghazy, MD, PhD, ${ }^{1,2}$ Marc Albert, MD, ${ }^{3}$ Henning Warnecke, MD, PhD, ${ }^{4}$ \\ Mirko Doss, MD, PhD,${ }^{5}$ Andreas Liebold, MD, PhD,${ }^{6}$ Edgar Eszlari, MD, PhD, ${ }^{7}$ Etem Caliskan, $\mathrm{MD},{ }^{8}$ \\ Taoufik Ouarrak, Dipl. Biol., ${ }^{9}$ Nicolas Doll, $\mathrm{MD}, \mathrm{PhD},{ }^{4,10}$ Jochen Senges, $\mathrm{MD}, \mathrm{PhD},{ }^{9}$ \\ Thorsten Hanke, MD, PhD, ${ }^{11}$ Ardawan J. Rastan, $\mathrm{MD}, \mathrm{PhD}^{1,2}$ \\ ${ }^{1}$ Department of Cardiac and Vascular Surgery, Herz-Kreislauf-Zentrum, Rotenburg, Germany; ${ }^{2}$ Department of Cardiac and Thoracic \\ Vascular Surgery, Philipps-University Hospital, Marburg, Germany; ${ }^{3}$ Department of Cardiac and Vascular Surgery, Robert-Bosch- \\ Krankenhaus, Stuttgart, Germany; ${ }^{4}$ Department of Cardiac Surgery, Schuechtermann-Klinik, Bad Rothenfelde, Germany; ${ }^{5}$ Department \\ of Cardiovascular Surgery, Kerckhoff Klinik, Bad Nauheim, Germany; ${ }^{6}$ Department of Cardiothoracic and Vascular Surgery, Ulm \\ University Hospital, Ulm, Germany; ${ }^{7}$ Department of Cardiovascular Surgery, Klinikum Bogenhausen, Munich, Germany; ${ }^{8}$ Department of \\ Cardiovascular Surgery, Charité Universitätsmedizin Berlin, Berlin, Germany; ${ }^{9}$ Stiftung Institut für Herzinfarktforschung, Ludwigshafen, \\ Germany; ${ }^{10}$ Department of Cardiac Surgery, Sana Heart Center, Stuttgart, Germany; ${ }^{11}$ Department for Cardiac Surgery, Asklepios \\ Klinikum Harburg, Hamburg, Germany
}

\section{ABSTRACT}

Background: Despite excellent data on lowering longterm stroke and all-cause mortality rates, currently, only $25-40 \%$ of atrial fibrillation (AF) patients undergo simultaneous surgical ablation therapy (SA) during cardiac surgery. Surgeon's fear exposing their patients to an additional, unjustified, and disproportionate risk when performing $\mathrm{SA}$ in $\mathrm{AF}$ patients presenting with sinus rhythm (SR) before surgery. To clarify the influence of preoperative SR before SA for AF, we conducted a subgroup analysis of the German Cardiosurgical Atrial Fibrillation (CASE-AF) register.

Methods: Between September 2016 and August 2020, $964 \mathrm{AF}$ patients with an underlying cardiac disease were scheduled for surgery with SA and enrolled in the CASE-AF register. Data prospectively were collected and analyzed retrospectively. We divided the entire cohort into an SR-group $(38.2 \%, \mathrm{~N}=368)$ and an AF-group $(61.8 \%, \mathrm{~N}=596)$, based on preoperative heart rhythm.

Results: Over half of the patients were moderately affected by their $\mathrm{AF}$, with no difference between the groups (European Heart Rhythm Association class $\geq$ IIb: SR-group 54.2\% versus $\mathrm{AF}$-group $58.5 \%, P=.238)$. The $\mathrm{AF}$-group had a higher preoperative EuroSCORE II $(4.8 \pm 8.0 \%$ versus $4.2 \pm 6.3 \%$, $P=.014$ ). In-hospital mortality (SR-group $0.8 \%$ versus AFgroup $1.7 \%, P=.261$ ), major perioperative adverse cardiac and cerebrovascular events (SR-group $2.7 \%$ versus $\mathrm{AF}$-group $3.5 \%, P=.500)$, and the new pacemaker implantation rate (SR-group $6.0 \%$ versus $\mathrm{AF}$-group $5.9 \%, P=.939$ ) were low

Received May 27, 2021; accepted fuly 27, 2021.

Correspondence: Maximilian Vondran, MD, Herz-Kreislauf-Zentrum Rotenburg, Department of Cardiac and Vascular Surgery, Heinz-Meise-Straße 100, 36199 Rotenburg, Germany; +49-6623-88-5858; fax: +49-6623-88-5860 (e-mail: m.vondran@hkz-rotenburg.de). and showed and no group difference. Logistic regression analysis showed a protective effect for preoperative SR to perioperative complications in $\mathrm{AF}$ patients undergoing $\mathrm{SA}$ (odds ratio (OR) 0.72 (95\% CI $0.52-0.998$ ); $P=.0485$ ).

Conclusions: Concomitant SA in AF patients presenting in SR before cardiac surgery is safe, has a low perioperative risk profile, and should be carried out with almost no exceptions.

\section{INTRODUCTION}

Depending on the underlying disease, $5-41 \%$ of patients who undergo cardiac surgery have a history of concomitant atrial fibrillation (AF) [Badhwar 2017]. Despite excellent data on an improved quality of life, lower long-term stroke rates and decreased all-cause mortality through surgical ablation therapy (SA) compared with non-treated $\mathrm{AF}$ patients [Badhwar 2017; Gillinov 2015; Iribarne 2019; Lee 2012; Musharbash 2018], as well as convincing guidelines [Badhwar 2017; Hindricks 2020; January 2019], currently, only 25-40\% of the AF patients were ablated simultaneously during cardiac surgery [Badhwar 2017]. Many cardiac surgeons seem to believe that AF patients in sinus rhythm (SR) at the time of hospital admission or surgery are unlikely to benefit from additional AF ablation. Moreover, some surgeons fear the risk of concomitant rhythm therapies, especially in patients presenting in SR at surgery with short-lasting paroxysmal AF carrying a low disease burden. Unfortunately, a temporary sinus rhythm with a symptom-free interval in a patient with accurately diagnosed $\mathrm{AF}$ is considered by some surgeons to constitute a sort of spontaneous cure. However, the heart's structural anomalies caused by AF persist despite the temporary sinus rhythm and will worsen as the $\mathrm{AF}$ progresses [Hindricks 2020].

There is a lack of data on the periprocedural safety of concomitant SA in patients with AF who present in SR before 
cardiac surgery. We therefore conducted a subgroup analysis of the nationwide, prospective, observational, multicenter German Cardiosurgical Atrial Fibrillation (CASE-AF) register to understand better the impact of a preoperative SR on the short-term safety outcome after SA. We expressly emphasize that this work's focus is not on investigating the effectiveness of SA to establish an SR.

\section{PATIENTS AND METHODS}

The (CASE-AF) is an on-going nationwide, prospective, observational, multicenter study governed by the Institute for Heart Attack Research (Institute für Herzinfarktforschung (IHF), Ludwigshafen, Germany) that collects data on the clinical outcome of patients undergoing SA for AF [Wehbe 2020]. Between September 2016 and August 2020, 17 German cardiac surgery centers enrolled 964 consecutive patients. Patients with AF and an underlying cardiac disease scheduled for surgery with concomitant SA or patients with stand-alone SA were included in the register. We divided the entire cohort into an SR group $(38.2 \%, \mathrm{~N}$ $=368)$ and AF-group (61.8\%, N = 596), in terms of their preoperative heart rhythm.

Study endpoints: In our study, the primary endpoint was all-cause in-hospital mortality. Secondary endpoints were stroke, myocardial infarction, new permanent pacemaker implantation (PPI), and the combined endpoint of major adverse cardiac and cerebrovascular events $(\mathrm{MACCE}=$ any death, myocardial infarction, and stroke). Additionally, we examined other major and heart-rhythm-specific outcome parameters during hospitalization and at the time of discharge.

Statistics: Categorical variables were presented as counts and percentages and were compared via the Chi-square test. Continuous variables were presented as median and interquartile-range or mean and standard deviation and compared by the Mann-Whitney-Wilcoxon test. Impact of preoperative SR on short-term outcomes was further tested in logistic regression analyses calculated as a comparison against the patients operated in AF in the CASE-AF register and corresponding odds ratio (OR) with $95 \%$ confidence interval (CI) were presented. All tests were 2 -tailed, and $P$-values $<.05$ were considered statistically significant. All analyses were performed using SAS statistical software package, version 9.4 (Cary, NC, USA).

Ethical considerations: This study was approved by the Ethics Committee (Landesärztekammer Rheinland-Pfalz, ID: 837.536 .15 [10304]). The CASE-AF register was entered into the ClinicalTrials.gov database (NCT03091452). The study design, pseudonymous data acquisition, and data publication follow the Declaration of Helsinki.

\section{RESULTS}

Table 1 summarizes preoperative patient characteristics. The SR-group's left ventricular ejection fraction (LVEF) was slightly higher $(55 \pm 11 \%$ versus $53 \pm 11 \%)$. Also, the
SR-group was more likely to have peripheral vascular disease $(7.6 \%$ versus $4.5 \%, P=.046)$, hepatic disease $(4.1 \%$ versus $1.5 \%$ ), and less likely to have chronic kidney disease (15.5\% versus $23.2 \%)$. Furthermore, the AF-group's EuroSCORE II was higher $(4.8 \pm 8.0 \%$ versus $4.2 \pm 6.3 \%)$. Moreover, AF-group patients were more likely to present a preoperative New York Heart Association class $\geq$ III $(57.2 \%$ versus $50.6 \%$ ). AF-group patients suffered from longer-lasting atrial fibrillation, but there was no difference in symptom severity between groups (European Heart Rhythm Association class $\geq \mathrm{IIb}$, SR-group $54.2 \%$ versus AF-group $58.5 \%)$. In addition, AF-group patients presented a negligibly larger left atrium $(49 \pm 10 \mathrm{~mm}$ versus 47 $\pm 9 \mathrm{~mm}$ ). As the leading underlying cardiac pathology, heart valve disease was more common in the AF-group than in the SR-group (64.1\% versus 54.6\%). Conversely, patients in the SR-group were more likely to have coronary artery disease $(37.4 \%$ versus $25.4 \%)$.

Table 2 illustrates periprocedural details. A minimally invasive approach to surgery was taken more often in the AF-group (36.0\% versus $26.2 \%, P=.003)$, and they underwent endocardial $(51.0 \%$ versus $43.9 \%, P=.031)$ and cryoablation $(52.0 \%$ versus $41.7 \%, P<.001)$ more frequently, whereas the SR-group underwent more epicardial $(64.0 \%$ versus $55.2 \%, P=.007)$ and radiofrequent $(58.3 \%$ versus $48.7 \%, P=.004)$ ablation. However, when the AF-group underwent radiofrequent ablation, the total duration of SA was longer than in the SR-group (468 $\pm 510 \mathrm{~s}$ versus 386 $\pm 483 \mathrm{~s}, P=.037)$. SA was more extensive in the AF group than the SR group. The proportion of box isolations $(71.0 \%$ versus $57.0 \%, P<.001)$ and application of LA lines $(50.4 \%$ versus $43.0 \%, P=.052)$ and RA lines $(15.0 \%$ versus $7.0 \%$, $P=.002)$ were more frequent in the AF-group. Immediately after surgery, more SR-group patients were in SR than in the AF-group (93.0\% versus $86.0 \%, P=.042)$.

Table 3 summarizes postoperative outcomes. The SRgroup's in-hospital mortality was below one percent; this value did not differ between groups (SR-group $0.8 \%$ versus $\mathrm{AF}$ group $1.7 \%, P=.261$ ). Major adverse cardiac and cerebrovascular events did not differ either between groups (SR-group $2.7 \%$ versus $\mathrm{AF}$-group $3.5 \%, P=.500$ ). We noted a higher rate of low cardiac output syndrome $(1.3 \%$ versus $0.0 \%, P=.026)$, severe postoperative bleeding $(3.5 \%$ versus $0.5 \%, P=.003)$, and re-thoracotomy ( $6.2 \%$ versus $1.9 \%, P=.002)$ in the $\mathrm{AF}$-group.

Table 4 displays SA-specific postoperative outcomes. The new PPI rate was six percent, which did not differ between groups (SR-group 6.0\% versus AF-group 5.9\%, $P=.939$ ). Twenty percent of the patients in both groups underwent cardioversion once postoperatively (SR-group $20.2 \%$ versus AF-group $18.5 \%, P=.513$ ). About three-quarters of the patients were taking a beta-blocker medication at discharge (SR-group 73.3\% versus $\mathrm{AF}$-group $75.9 \%, P=.361$ ). Less than $90 \%$ were orally anticoagulated (SR-group $87.7 \%$ versus $\mathrm{AF}$-group $87.9 \%, P=.948$ ), a value that did not differ between groups. However, the AF-group's proportion of class III antiarrhythmic amiodarone (discharge medication) was lower $(26.1 \%$ versus $35.7 \%, P=.002)$. Furthermore, the AF-group was more likely to be taking digitalis glycoside 
upon discharge ( $4.0 \%$ versus $1.6 \%, P=.037)$. At discharge, $81.3 \%$ of the SR-group and $61.2 \%$ of the AF-group had sinus rhythm $(P<.001)$.

Logistic regression analysis showed a protective effect on perioperative complications (combined endpoint of all complications listed in Table 3 and 4) for the SR before cardiac surgery with SA (odds ratio (OR) 0.72 (95\% CI 0.52 0.998); $P=.0485$ ). Also, patients with preoperative SR were almost three times more likely to be discharged in sinus rhythm (adjusted OR 2.81 (95\% CI 2.05-3.84); $P$ <.0001).

Table 1. Preoperative patient characteristics

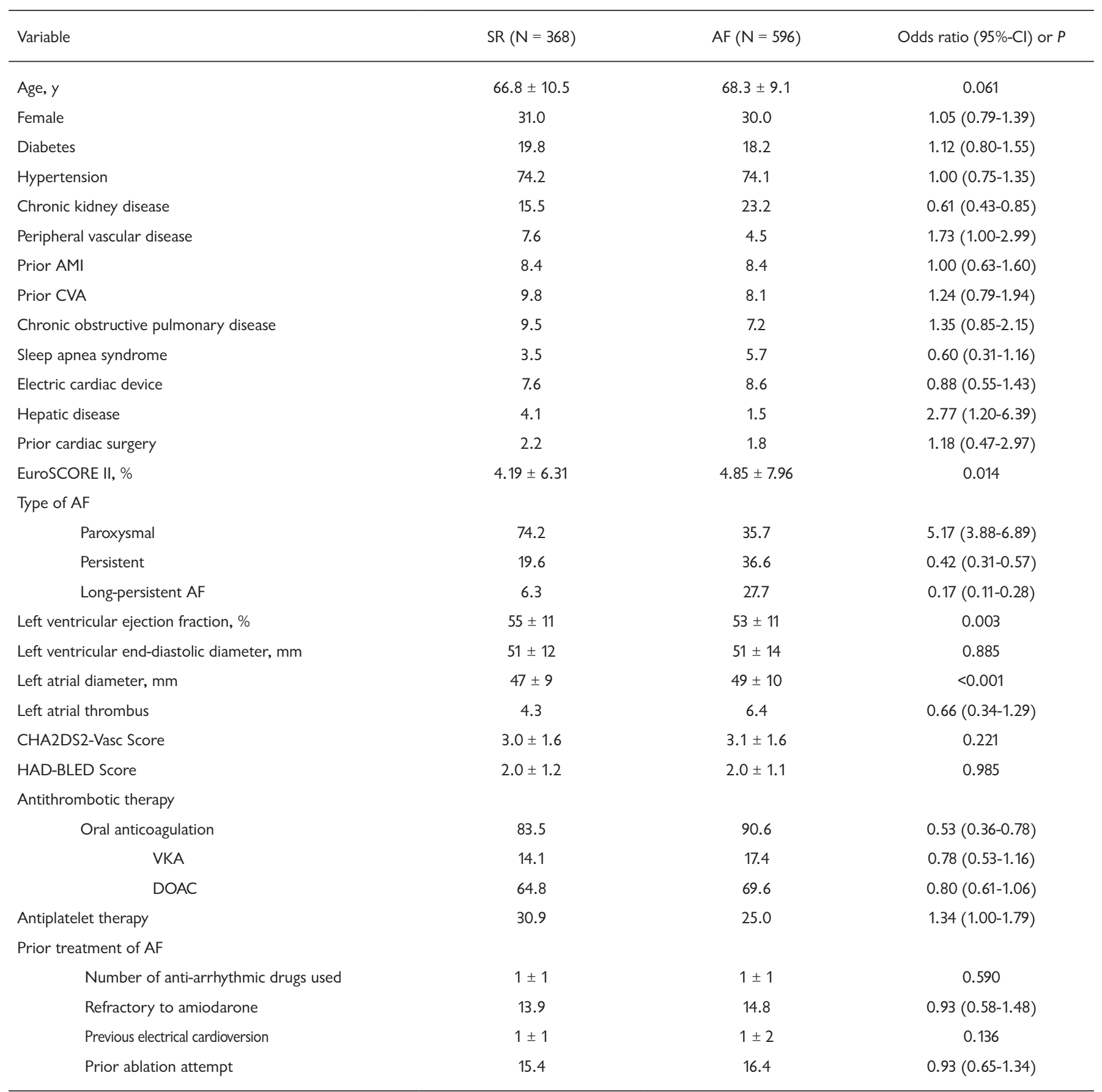

AF, atrial fibrillation; AMI, acute myocardial infarction; CVA, cerebrovascular accident; DOAC, direct oral anticoagulants; SR, sinus rhythm; VKA, vitamin K antagonist 
Table 2. Periprocedural details

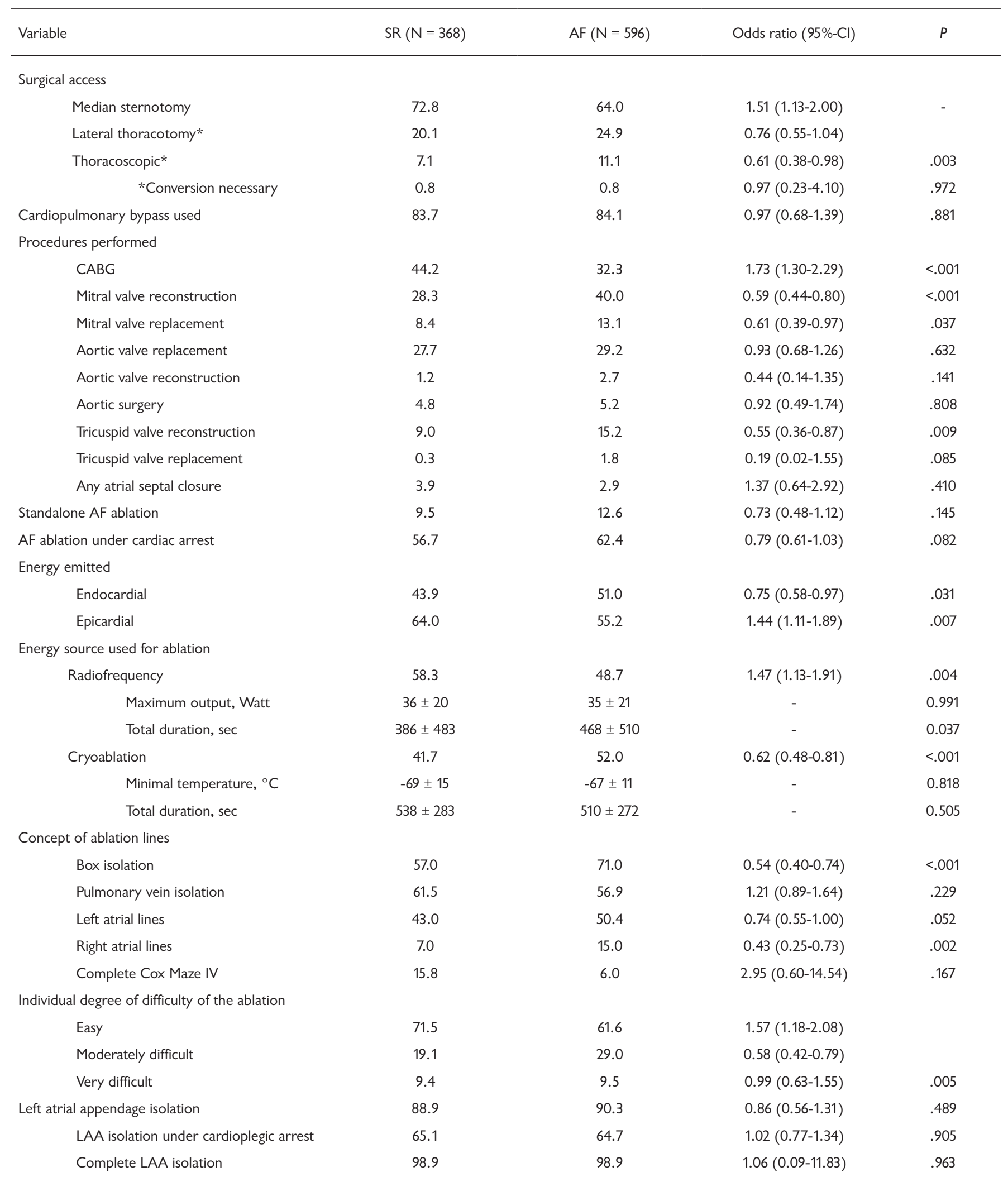


Table 2. Periprocedural details [CONT.]

\begin{tabular}{lcccc}
\hline \multicolumn{1}{l}{ Variable } & SR $(\mathrm{N}=368)$ & AF $(\mathrm{N}=596)$ & Odds ratio $(95 \%-\mathrm{Cl})$ \\
\hline Sinus rhythm & 93.0 & 86.6 & $2.07(1.01-4.23)$ \\
Atrial tachycardia & 1.3 & 8.1 & $0.14(0.03-0.63)$ & .042 \\
Atrioventricular block 2nd degree & 1.3 & 1.6 & $0.78(0.14-4.29)$ & .003 \\
Atrioventricular block 3rd degree & 4.4 & 3.7 & $1.22(0.45-3.35)$ & .699 \\
\hline
\end{tabular}

AF, atrial fibrillation; $C A B G$, coronary artery bypass grafting; $\mathrm{Cl}$, confidence interval; LAA, left atrial appendage; OR, odds ratio; SR, sinus rhythm

\section{DISCUSSION}

Our findings can be summarized as: Additional SA during cardiac surgery in patients with known AF who present for surgery in SR is safe. These patients were not exposed to an additional perioperative risk of mortality and morbidity. We found that the presence of preoperative SR had a protective effect, that is, fewer perioperative complications, in patients with $\mathrm{AF}$ who underwent concomitant SA.

The frequency of SA in AF patients undergoing cardiac surgery is relatively low [Badhwar 2017; McCarthy 2020]. Unfortunately, there is almost no data on surgeons' reasons for avoiding SA. AF patients with SR before surgery probably represent part of the non-ablated patient group. Some surgeons assume that by performing an $\mathrm{SA}$ in $\mathrm{AF}$ patients with preoperative SR, they are unjustifiably increasing mortality and morbidity with no benefit to their patients, especially when confronting very brief or permanent AF. However, SA is most successful in patients with short-lasting atrial fibrillation, when the left atrium diameter is still small, and when the patient is younger [Beukema 2008; Chaiyaroj 2008; Chen 2004; Gillinov 2006; Sunderland 2011]. Especially in short-lasting AF cases, it is very rewarding to carry out an additional ablation at an early stage of pending heart surgery to re-establish SR permanently.

In-hospital mortality was surprisingly low in our study. With an in-hospital mortality rate of around $4.5 \%$ calculated preoperatively via the EuroSCORE II, only $0.8 \%$ of our SR-group patients died in hospital, despite simultaneous SA. Note that the in-hospital mortality rate of patients presenting with AF before surgery in the CASE-AF register was $1.2 \%$. It is common knowledge that mortality among heart surgery patients rises in conjunction with an intervention's increasing complexity caused by their underlying pathology, more extensive surgery, and longer cardiopulmonary bypass times [Iino 2017; Salis 2008]. If we compare our SRgroup patients' in-hospital mortality $(0.8 \%)$ with data from recent large, randomized controlled trials (RCT) on bypass surgery or aortic valve surgery (where in-hospital mortality was 1.0-3.5\% [Mack 2019; Mäkikallio 2016; Popma 2019; Serruys 2009; Stone 2016], our real-world registry results match those of patients who underwent only isolated cardiac procedures within large and highly selective RCTs without additional SA. Moreover, we observe the same for stroke and
MACCE [Mack 2019; Mäkikallio 2016; Popma 2019; Serruys 2009; Stone 2016]. Also, no patient in our SR-group suffered a complication, which are more likely to occur in interventional ablations (where it is around $0.5 \%$, i.e., pulmonary vein injury or stenosis, phrenic nerve palsy, vena cava, or esophageal injury [Kany 2021; Rottner 2021; Zylla 2020]). In addition, only $0.3 \%$ of patients in the SR group had a pulmonary embolism. Additional SA increases perioperative mortality and morbidity neither in patients with any $\mathrm{AF}$, as research groups have recently reported [Ad 2012; Gillinov 2015; Lee 2012; Malaisrie 2012; Musharbash 2018], nor in patients with paroxysmal AF exclusively [McCarthy 2013]. Moreover, there is evidence of no difference in perioperative mortality and morbidity compared with patients without $\mathrm{AF}$ who did not undergo additional SA [Ad 2012; Lee 2012; Musharbash 2018]. In fact, there is recent evidence of a long-term survival benefit for patients with SA compared with non-ablated AF patients [Iribarne 2019; Lee 2012; Musharbash 2018].

The low rate of perioperative stroke $(1.6 \%)$ in our study and in the entire CASE-AF register cohort might also be due to the high number of additional left atrial appendage (LAA) isolations. Our approximately $90 \%$ LAA isolation rate is impressive, but there is still room for improvement. A recent paper relying on the Society of Thoracic Surgeons Adult Cardiac Surgery Database examined nearly 11,000 AF patients undergoing cardiac surgery [Friedman 2018]. Their LAA isolation rate was only $37 \%$. However, the rate of additional SA unfortunately was only about $42 \%$. In patients with additional SA, the rate of LAA isolation was 94\% - somewhat similar to our data [Friedman 2018]. Moreover, in a meta-analysis by Tsai et al. involving a total of 3,653 cardiac surgery patients undergoing SA, the group with additional LAA isolation suffered significantly fewer perioperative strokes and no increased mortality or higher reoperation rates due to bleeding [Tsai 2015]. Cox and colleagues also demonstrated that resection or complete closure of the LAA significantly reduced the perioperative stroke risk, and nearly eliminated the long-term stroke risk [Cox 1999]. We emphasize that LAA isolation must be done right: The most effective closure options are excision [Kanderian 2008], which can only be applied by open-heart surgery, and accessindependent isolation of the LAA utilizing a clip [Emmert 2014]. Furthermore, the closure technique should be adapted 
Table 3. Overall postoperative outcomes

\begin{tabular}{|c|c|c|c|c|}
\hline In-hospital mortality & 0.8 & 1.7 & $0.48(0.13-1.77)$ & .261 \\
\hline Cerebrovascular accident & 1.6 & 1.5 & $1.08(0.38-3.07)$ & .879 \\
\hline MACCE & 2.7 & 3.5 & $0.77(0.36-1.65)$ & .500 \\
\hline Low cardiac output & 0.0 & 1.3 & - & .026 \\
\hline Acute kidney injury & 1.1 & 2.7 & $0.40(0.13-1.20)$ & .092 \\
\hline Pulmonary embolism & 0.3 & 0 & - & .202 \\
\hline Respiratory failure & 0.8 & 2.5 & $0.32(0.09-1.11)$ & .059 \\
\hline $\mathrm{TIA}<24 \mathrm{~h}$ & 1.1 & 0.5 & $2.18(0.48-9.79)$ & .298 \\
\hline Atrioventricular dissection & 0 & 0.2 & - & .432 \\
\hline Injury of the inferior vena cava & 0 & 0.2 & - & .432 \\
\hline Injury of the ramus circumflexus & 0.3 & 0 & - & .202 \\
\hline Pulmonary vein injury & 0.0 & 0.2 & - & .432 \\
\hline Esophagus injury & 0.0 & 0.0 & - & - \\
\hline Severe post-op bleeding & 0.5 & 3.5 & $0.15(0.03-0.64)$ & .003 \\
\hline Re-thoracotomy & 1.9 & 6.2 & $0.29(0.13-0.67)$ & .002 \\
\hline Redo surgery & 0.3 & 0.3 & $0.81(0.07-8.98)$ & .865 \\
\hline Deep sternal wound infection & 0.5 & 0.8 & $0.65(0.13-3.36)$ & .602 \\
\hline
\end{tabular}

AF, atrial fibrillation; $\mathrm{Cl}$, confidence interval; CPR, cardiopulmonary resuscitation; MACCE, major adverse cardiac and cerebrovascular events; OR, odds ratio;

$\mathrm{SR}$, sinus rhythm; TIA, transient ischemic attack

to the LAA's anatomy, and residual LAA perfusion should be avoided as much as possible, since that raises the risk of thrombus formation [Di Biase 2012]. To ensure complete LAA isolation without residual perfusion and avoid creating iatrogenic stroke sources, we recommend intraoperative TOE monitoring. TOE should be repeated after three months.

A further argument against performing simultaneous SA in subjectively unsuitable $\mathrm{AF}$ patients is the higher rate of new PPI, because permanent right ventricular pacing triggers an increased incidence of $\mathrm{AF}$, heart failure, and mortality [Nielsen 2003; Sweeney 2003; Wilkoff 2002]. After SA, the PPI rates in the literature range from 0.8 to $23.3 \%$ [Churyla 2020]. In our work, the rate of new in-hospital PPI was $6.5 \%$. In the study of Badhwar et al. [Badhwar 2017], the rate was 7.6\%, and in Musharbash et al. [Musharbash 2018], even 11.0\%. Nevertheless, both studies demonstrated a long-term survival advantage for the ablated AF patients compared with the non-ablated group [Badhwar 2017; Musharbash 2018]. However, the reasons for requiring a PPI after SA are not yet well understood. A junctional rhythm and sick-sinus-syndrome that is demasked after SA are possible contributing negative factors [Cox 2018]. The junctional rhythm usually recedes and is often worsened by faulty surgical manipulation [Cox 2018]. Due to the understandable reluctance to discharge patients in junctional rhythm and the financial pressures of keeping in-hospital stays brief, many patients unnecessarily receive a PPI. As already proven by Cox and colleagues in 1993, patients with a sufficiently functioning sinoatrial node do not need a PPI after SA [Cox 1993]. The SA itself probably plays a minor role in a PPI's necessity later; surgical technique and experience seem to play a much more significant role [Churyla 2020].

Limitations: This study's most important limitation is that only patients with AF were included in the CASE-AFregister. Establishing a control group for comparison with non-ablated patients in SR is not possible with our registry data. Also, our results reflect only the short-term outcomes and do not include a long-term follow up. Furthermore, we explicitly state again that this study was not designed to investigate SA efficacy. Instead, we wanted to investigate 
Table 4. AF-ablation-specific postoperative outcomes

\begin{tabular}{|c|c|c|c|c|}
\hline \multicolumn{5}{|l|}{ Severe cardiac arrhythmias after surgery } \\
\hline AV block 3rd degree & 4.4 & 4.5 & $0.96(0.51-1.81)$ & .901 \\
\hline Sinoatrial arrest & 1.6 & 1.2 & $1.40(0.47-4.19)$ & .548 \\
\hline Pharmaceutical & 32.9 & 21.1 & $1.83(0.94-3.58)$ & .060 \\
\hline Number of cardioversions & $1 \pm 1(N=74)$ & $1 \pm 1(\mathrm{~N}=110)$ & - & .016 \\
\hline New pacemaker implantation & 6.0 & 5.9 & $1.02(0.59-1.77)$ & .939 \\
\hline \multicolumn{5}{|l|}{ Medication at discharge } \\
\hline \multicolumn{5}{|l|}{ Antiarrhythmic drugs } \\
\hline Class III & 35.7 & 26.1 & $1.57(1.19-2.08)$ & .002 \\
\hline Digitalis & 1.6 & 4.0 & $0.39(0.16-0.98)$ & .037 \\
\hline Anticoagulation & 87.7 & 87.9 & $0.99(0.66-1.47)$ & .948 \\
\hline VKA & 41.9 & 44.6 & $0.90(0.68-1.19)$ & .440 \\
\hline UFH & 2.2 & 2.1 & $1.03(0.40-2.69)$ & .948 \\
\hline LMWH & 12.7 & 16.3 & $0.75(0.50-1.12)$ & .160 \\
\hline DOAC & 46.0 & 42.3 & $1.17(0.90-1.52)$ & .249 \\
\hline Anti-platelet therapy & 53.4 & 47.5 & $1.27(0.98-1.65)$ & .074 \\
\hline ACE inhibitor/ARB & 46.0 & 49.8 & $0.86(0.66-1.12)$ & .254 \\
\hline Atrial fibrillation & 14.1 & 31.7 & $0.35(0.25-0.50)$ & \\
\hline Other & 4.6 & 7.0 & $0.64(0.36-1.14)$ & $<.001$ \\
\hline
\end{tabular}

ACE, Angiotensin-converting enzyme; AF, atrial fibrillation; ARB, angiotensin receptor blocker; DOAC, direct oral anticoagulants; NSAID, LMWH, Low molecular weight heparin; Nonsteroidal anti-inflammatory drugs; SR, sinus rhythm, UFH, Unfractionated heparin; VKA, vitamin K antagonist

perioperative patient safety and the adverse event profile in AF patients with preoperative SR who underwent additional SA. Most of this study's other limitations lie in its observational and retrospective design.

\section{CONCLUSION}

Simultaneous SA is safe in patients with known AF presenting in SR for cardiac surgery and carries a low risk for perioperative mortality and morbidity. However, depending on the index procedure performed, a slightly higher new PPI rate may appear if concomitant SA is performed. Our data revealed no justification for avoiding concomitant SA during heart surgery in AF patients with a preoperative SR. On the contrary, there is evidence of SA's protective perioperative effect in these patients. To better understand the impact of preoperative SR on the safety and efficacy of SA in AF patients, prospective randomized trials are warranted. 


\section{ACKNOWLEDGEMENT}

The authors wish to thank Ms. Belgin Özdemir for providing excellent administrative support.

Availability of data and materials: The anonymized data used to support this study's findings may be released upon application to Ms. Belgin Özdemir (IHF, Ludwigshafen, Germany), who can be contacted at oezdemir(at)stiftung-ihf.de.

Funding: The CASE-AF registry is supported by a grant from Atricure Europe BV, De Entree 260, 1101 EE Amsterdam Z. O.

\section{REFERENCES}

Ad N, Henry L, Hunt S, Holmes SD. 2012. Do we increase the operative risk by adding the cox maze iii procedure to aortic valve replacement and coronary artery bypass surgery? The Journal of Thoracic and Cardiovascular Surgery. 143(4):936-944.

Badhwar V, Rankin JS, Ad N, et al. 2017. Surgical ablation of atrial fibrillation in the united states: Trends and propensity matched outcomes. The Annals of Thoracic Surgery. 104(2):493-500.

Badhwar V, Rankin JS, Damiano Jr RJ, et al. 2017. The Society of Thoracic Surgeons 2017 clinical practice guidelines for the surgical treatment of atrial fibrillation. The Annals of Thoracic Surgery. 103(1):329-341.

Beukema WP, Sie HT, Misier AR, Delnoy PP, Wellens HJ, Elvan A. 2008. Predictive factors of sustained sinus rhythm and recurrent atrial fibrillation after a radiofrequency modified maze procedure. European journal of cardio-thoracic surgery : official journal of the European Association for Cardio-thoracic Surgery. 34(4):771-775.

Chaiyaroj S, Ngarmukos T, Lertsithichai P. 2008. Predictors of sinus rhythm after radiofrequency maze and mitral valve surgery. Asian Cardiovascular \& Thoracic Annals. 16(4):292-297.

Chen MC, Chang JP, Chang HW. 2004. Preoperative atrial size predicts the success of radiofrequency maze procedure for permanent atrial fibrillation in patients undergoing concomitant valvular surgery. Chest. 125(6):2129-2134.

Churyla A, Desai A, Kruse J, Cox J, McCarthy P. 2020. Concomitant atrial fibrillation ablation in patients undergoing coronary artery bypass and cardiac valve surgery. Journal of Cardiovascular Electrophysiology.

Cox JL, Ad N, Churyla A, et al. 2018. The maze procedure and postoperative pacemakers. The Annals of Thoracic Surgery. 106(5):1561-1569.

Cox JL, Ad N, Palazzo T. 1999. Impact of the maze procedure on the stroke rate in patients with atrial fibrillation. The Journal of Thoracic and Cardiovascular Surgery. 118(5):833-840.

Cox JL, Boineau JP, Schuessler RB, Kater KM, Lappas DG. 1993. Fiveyear experience with the maze procedure for atrial fibrillation. The Annals of Thoracic Surgery. 56(4):814-823; discussion 823-814.

Di Biase L, Santangeli P, Anselmino M, et al. 2012. Does the left atrial appendage morphology correlate with the risk of stroke in patients with atrial fibrillation? Results from a multicenter study. Journal of the American College of Cardiology. 60(6):531-538.

Emmert MY, Puippe G, Baumüller S, et al. 2014. Safe, effective and durable epicardial left atrial appendage clip occlusion in patients with atrial fibrillation undergoing cardiac surgery: First long-term results from a prospective device trial. European Journal of Cardio-Thoracic Surgery. 45(1):126-131.
Friedman DJ, Piccini JP, Wang T, et al. 2018. Association between left atrial appendage occlusion and readmission for thromboembolism among patients with atrial fibrillation undergoing concomitant cardiac surgery. Jama. 319(4):365-374.

Gillinov AM, Bhavani S, Blackstone EH, et al. 2006. Surgery for permanent atrial fibrillation: Impact of patient factors and lesion set. The Annals of Thoracic Surgery. 82(2):502-513; discussion 513-504.

Gillinov AM, Gelijns AC, Parides MK, et al. 2015. Surgical ablation of atrial fibrillation during mitral-valve surgery. The New England Journal of Medicine. 372(15):1399-1409.

Hindricks G, Potpara T, Dagres N, et al. 2020. 2020 esc guidelines for the diagnosis and management of atrial fibrillation developed in collaboration with the european association of cardio-thoracic surgery (eacts). European Heart Journal.

Iino K, Miyata H, Motomura N, et al. 2017. Prolonged cross-clamping during aortic valve replacement is an independent predictor of postoperative morbidity and mortality: Analysis of the japan cardiovascular surgery database. The Annals of Thoracic Surgery. 103(2):602-609.

Iribarne A, DiScipio AW, McCullough JN, et al. 2019. Surgical atrial fibrillation ablation improves long-term survival: A multicenter analysis. The Annals of Thoracic Surgery. 107(1):135-142.

January CT, Wann LS, Calkins H, et al. 2019. 2019 aha/acc/hrs focused update of the $2014 \mathrm{aha} / \mathrm{acc} / \mathrm{hrs}$ guideline for the management of patients with atrial fibrillation: A report of the american college of cardiology/ american heart association task force on clinical practice guidelines and the heart rhythm society in collaboration with the society of thoracic surgeons. Circulation. 140(2):e125-e151.

Kanderian AS, Gillinov AM, Pettersson GB, Blackstone E, Klein AL. 2008. Success of surgical left atrial appendage closure: Assessment by transesophageal echocardiography. Journal of the American College of Cardiology. 52(11):924-929.

Kany S, Brachmann J, Lewalter T, et al. 2021. Safety and patient-reported outcomes in index ablation versus repeat ablation in atrial fibrillation: Insights from the german ablation registry. Clinical research in cardiology : official journal of the German Cardiac Society. 110(6):841-850.

Lee R, McCarthy PM, Wang EC, et al. 2012. Midterm survival in patients treated for atrial fibrillation: A propensity-matched comparison to patients without a history of atrial fibrillation. The Journal of Thoracic and Cardiovascular Surgery. 143(6):1341-1351; discussion 1350-1341.

Mack MJ, Leon MB, Thourani VH, et al. 2019. Transcatheter aorticvalve replacement with a balloon-expandable valve in low-risk patients. The New England Journal of Medicine. 380(18):1695-1705.

Mäkikallio T, Holm NR, Lindsay M, et al. 2016. Percutaneous coronary angioplasty versus coronary artery bypass grafting in treatment of unprotected left main stenosis (noble): A prospective, randomised, open-label, non-inferiority trial. Lancet (London, England). 388(10061):2743-2752.

Malaisrie SC, Lee R, Kruse J, et al. 2012. Atrial fibrillation ablation in patients undergoing aortic valve replacement. The Journal of Heart Valve Disease. 21(3):350-357.

McCarthy PM, Davidson CJ, Kruse J, et al. 2020. Prevalence of atrial fibrillation before cardiac surgery and factors associated with concomitant ablation. The Journal of Thoracic and Cardiovascular Surgery. 159(6):2245-2253.e2215.

McCarthy PM, Manjunath A, Kruse J, et al. 2013. Should paroxysmal atrial fibrillation be treated during cardiac surgery? The Journal of Thoracic and Cardiovascular Surgery. 146(4):810-823. 
Musharbash FN, Schill MR, Sinn LA, et al. 2018. Performance of the cox-maze iv procedure is associated with improved long-term survival in patients with atrial fibrillation undergoing cardiac surgery. The Journal of Thoracic and Cardiovascular Surgery. 155(1):159-170.

Nielsen JC, Kristensen L, Andersen HR, Mortensen PT, Pedersen OL, Pedersen AK. 2003. A randomized comparison of atrial and dual-chamber pacing in 177 consecutive patients with sick sinus syndrome: Echocardiographic and clinical outcome. Journal of the American College of Cardiology. 42(4):614-623.

Popma JJ, Deeb GM, Yakubov SJ, et al. 2019. Transcatheter aortic-valve replacement with a self-expanding valve in low-risk patients. The New England Journal of Medicine. 380(18):1706-1715.

Rottner L, Brachmann J, Lewalter T, et al. 2021. The impact of basic atrial rhythm during catheter ablation of atrial fibrillation on clinical outcomes: Lessons from the german ablation registry. Journal of Cardiovascular Electrophysiology. 32(7):1833-1841.

Salis S, Mazzanti VV, Merli G, et al. 2008. Cardiopulmonary bypass duration is an independent predictor of morbidity and mortality after cardiac surgery. Journal of Cardiothoracic and Vascular Anesthesia. 22(6):814-822.

Serruys PW, Morice MC, Kappetein AP, et al. 2009. Percutaneous coronary intervention versus coronary-artery bypass grafting for severe coronary artery disease. The New England Journal of Medicine. 360(10):961-972.
Stone GW, Sabik JF, Serruys PW, et al. 2016. Everolimus-eluting stents or bypass surgery for left main coronary artery disease. The New England Journal of Medicine. 375(23):2223-2235.

Sunderland N, Maruthappu M, Nagendran M. 2011. What size of left atrium significantly impairs the success of maze surgery for atrial fibrillation? Interactive cardiovascular and thoracic surgery 13(3):332-338.

Sweeney MO, Hellkamp AS, Ellenbogen KA, et al. 2003. Adverse effect of ventricular pacing on heart failure and atrial fibrillation among patients with normal baseline qrs duration in a clinical trial of pacemaker therapy for sinus node dysfunction. Circulation. 107(23):2932-2937.

Tsai Y-C, Phan K, Munkholm-Larsen S, Tian DH, La Meir M, Yan TD. 2015. Surgical left atrial appendage occlusion during cardiac surgery for patients with atrial fibrillation: A meta-analysis. European Journal of Cardio-Thoracic Surgery. 47(5):847-854.

Wehbe M, Albert M, Warnecke H, et al. 2020. V423 - the german cardiosurgical atrial fibrillation registry (case-af): In-hospital outcomes. Clinical Research in Cardiology. p. 1-1.

Wilkoff BL, Cook JR, Epstein AE, et al. 2002. Dual-chamber pacing or ventricular backup pacing in patients with an implantable defibrillator: The dual chamber and vvi implantable defibrillator (david) trial. Jama. 288(24):3115-3123

Zylla MM, Hochadel M, Andresen D, et al. 2020. Ablation of atrial fibrillation in patients with hypertension-an analysis from the german ablation registry. Journal of Clinical Medicine. 9(8). 